\title{
COVID-19: Was wir wissen können
}

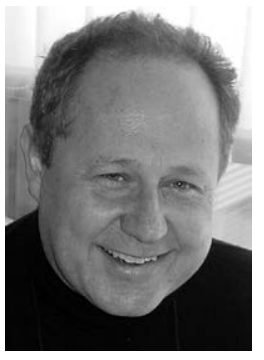

Manfred Wildner
Bibliografie

DOI https://doi.org/10.1055/a-1174-5692

Gesundheitswesen 2020; 82: 493-496

(c) Georg Thieme Verlag KG Stuttgart · New York

ISSN 0941-3790

Korrespondenzadresse

Prof. Dr. med. Manfred Wildner

Bayerisches Landesamt für Gesundheit und Lebensmittelsicherheit

Veterinärstraße 2

85764 Oberschleißheim

Manfred.Wildner@|gl.bayern.de
Die Ausarbeitung seiner Logik-Vorlesungen hatte Immanuel Kant an Gottlob Benjamin Jäsche übertragen, welcher im Jahr 1800 ein Handbuch dazu veröffentlichte. Dieser Niederschrift verdanken wir die einprägsame Formulierung: „Das Feld der Philosophie [...] läßt sich auffolgende Fragen bringen: 1) Was kann ich wissen? - 2) Was soll ich tun? 3) Was darf ich hoffen? 4) Was ist der Mensch?" [1]. Diese Frage nach dem berechtigten Wissen-Können hat nicht nur Immanuel Kant und seine Zeitgenossen beschäftigt, sondern ist eine Kernfrage von den Anfängen der Philosophie bis zu modernen heutigen Wissenschaftstheorien [2]. Die damit verbundene Skepsis exemplifiziert durch den Empirismus David Humes - unterbrach nach eigenen Angaben Immanuel Kants seinen „dogmatischen Schlummer“ und gab seinem Denken „eine ganz andere Richtung“ [3].

Skepsis in Abgrenzung zu Dogmatik ist heute geradezu eine Vorbedingung moderner Wissenschaftlichkeit. Nicht im Sinne des unbegründeten Zweifels, sondern als systematisches Hinterfragen dessen, was wir erkennen und wissen können und als berechtigte und notwendige Frage nach den Grenzen von Wissen - und wohl auch des Nichtwissens. Eine Eingrenzung dieser grundsätzlichen Skepsis geschieht u. a. in einem Wechselspiel von empirisch erfahrbaren Messungen und Eindrücken mit einer logischen, vernünftigen a priori bestehenden Ordnung und Interpretation dieser Eindrücke. Wissenschaft ist dabei immer zukunftsoffen und niemals abgeschlossen - was heute gilt, kann morgen schon überholt sein. Dem Statistiker George Box wird ein dieses Dilemma pointierender Aphorismus zugeschrieben: „All models are wrong, but some are useful“ [4]. Als Schritt zur Lösung dieses Dilemmas schlägt er ein iteratives gegenseitiges Feedback von Theorie und Praxis vor, neben dem Ockhamschen Sparsamkeitsprinzip, der Bereitschaft, aus Fehlern zulernen, einer Differenzierung zwischen Wichtigem und Unwichtigem (selective worrying) und der Einordnung der Mathematik als v. a. nützliches Instrument zur Beschreibung der Wirklichkeit.

Nur Überlegungen aus dem Elfenbeinturm der Wissenschaft, weit entfernt von den tatsächlichen Problemen der Praxis, wie sie sich ganz konkret im Zusammenhang mit der COVID-19-Pandemie stellen? Ganz im Gegenteil - eine auch praktische Nützlichkeit zeigt sich in der Anwendung auf diese konkrete Herausforderung von großem Ernst. Die Fragen, welche sich im Zusammenhang mit dem SARS-CoV-2-Virus und der von ihm ausgelösten Krankheit COVID19 stellen, sind vielfältig. Sie betreffen den Ursprung dieses Geschehens als ätiologische Fragestellung, die Auswirkungen auf die Gesundheit infizierter Menschen als pathogenetische und klinische Fragestellung, die bevölkerungsmedizinische Dynamik als (infektions-) epidemiologische Fragestellung, die Möglichkeiten des Infektionsschutzes und die damit verbundenen materiellen und nicht-materiellen Kosten und Risiken in einem Ineinandergreifen von Wissenschaft und Praxis als Public-Health-Fragestellung und darüber hinaus vielfältige Fragen aus einer gesellschaftlichen, auch gesellschaftspolitischen Perspektive. Nicht zuletzt wird auch die Frage gestellt, ob diese Pandemie denn real sei oder womöglich nur eine interessensgeleitete mediale Inszenierung.

Jede dieser Fragestellungen ist berechtigt und jede ist auf zeitnahe und auch zeitnah angemessen finanzierte weitere Forschungen angewiesen. Unser Erkenntnisbedarf in den Grundlagenwissenschaften, in Prävention und Epidemiologie, Klinik und Versorgungsforschung, Public Health und Politik ist groß, Entscheidungen drängen und können derzeit oft nur unter großer Unsicherheit getroffen werden. Dies betrifft alle Entscheidungsträger in Politik und Gesundheitswesen und in einer offenen Gesellschaft auch die einzelnen Menschen. Ein kleines, nicht abschließendes Inventar des wie immer vorläufigen Wissens soll in diesem Zusammenhang orientierend und gleichzeitig auch exemplarisch für die Zukunft versucht werden.

Ätiologie Wir kennen als Erreger das SARS-CoV-2-Virus, ein bisher nicht bekanntes zoonotisches behülltes RNA-Virus, dessen nächste Verwandte in einer genomischen Analyse bei Fledermäusen in China isolierte und sequenzierte Viren sind [5]. Die gesicherte Identifizierung eines Zwischenwirtes für die vermutete Übertragung auf den Menschen auf einem wet market in Wuhan ist bisher nicht erfolgt, was zu Spekulationen über eine mögliche Freisetzung 
aus einem dortigen Hochsicherheitslabor geführt hat - nicht zuletzt auch zu einem politischen Schlagabtausch („chinese virus“). Pathogenese Noch offen sind die individualmedizinischen Krankheitsverläufe und Organbeteiligungen bei infizierten und erkrankten Personen. Anders als die auf spezifische Enzyme der Lungenzellen angewiesenen Influenzaviren zeigt sich bei den schweren COVID-19 Verläufen eine Betroffenheit verschiedenster Organe (Multiorganversagen), welche auf eine Infektion des Gefäßendothels mittels des weit verbreiteten ACE-2-Rezeptors zurückgeführt werden könnte [6]. Eine weitere Absicherung dieser Erkenntnis, z. B. durch Autopsiestudien, hätte Auswirkungen auf Therapie und Prognose.

Epidemiologie und Prävention Immunologische Untersuchungen der ersten Fallserie in Deutschland deuten auf eine wesentliche a- und präsymptomatische Infektiösität sowie eine möglicherweise unvollständige Immunität im weiteren Verlauf hin - Erkenntnisse, welche Konsequenzen für präventive Maßnahmen wie die Bestimmung der relevanten Kontakte für den bevölkerungsbezogenen Infektionsschutz und auch für die Erfolgsaussichten von Impfstrategien haben [7]. Relevante Erkenntnisdefizite bestehen auch noch bei den klinisch-epidemiologischen Verlaufsbeschreibungen, bei der Festlegung der infektionsepidemiologischen Kennzahlen und ihren versorgungsepidemiologischen Korrelaten sowie im Feld der Risikofaktor-bezogenen Umweltepidemiologie (z. B. Übertragung durch Aerosole). Die Unzuverlässigkeit von frühen Berechnungen der sog. Case Fatality Rate (CFR) sind bezüglich der Influenza hinlänglich beschrieben worden [8] und auch für COVID-19 thematisiert [9]. Die Letalität unter Erkrankten ist etwas anderes als die Sterblichkeit der oft unterschiedlich definierten „Fälle“ der CFR, welche Krankenhausfälle, Intensivpatienten, Meldefälle u.a.m. sein können. Empfohlen wird daher die explizite Angabe der Sterblichkeit unter Hospitalpatienten (HFR) oder unter Infizierten (IFR) [8]. Eine „naive“ Letalität aus aktuell gemeldeten Infizierten und aktuell gemeldeten Todesfällen zu errechnen, ist wenig hilfreich: während Todesfälle weitgehend vollständig erfasst werden, schwankt die Untererfassung von Infizierten durch die Meldesysteme unter Pandemiebedingungen im Zeitverlauf und zwischen Ländern und kann allein dadurch die Anzahl der tatsächlich Infizierten um den Faktor 10 oder mehr unterschätzen [10]. Hinzu kommen Aspekte der zeitlichen Zuordenbarkeit - zu den nicht-trivialen methodischen Herausforderungen siehe Ghani et al. [11].

Die Letalität unter Erkrankten und Infizierten kann derzeit ebenso wie der Anteil asymptomatisch Infizierter nur aus Quasi-Experimenten wie der vollständigen Untersuchung der Passagiere von Kreuzfahrtschiffen oder im Flugverkehr im Rahmen von Reiserückholaktionen geschätzt werden [10] sowie aus den noch viel zu seltenen gründlichen Aufarbeitungen regionaler Ausbrüche [12]. Realistisch scheint die Annahme von einem Viertel bis zur Hälfte asymptomatischer Fälle sowie eine bevölkerungsbezogene Letalität von etwa $1 \%$ der Erkrankten [9] bzw. etwa 0,5\% der Infizierten. Damit liegt die COVID-19-Letalität bei einem Mehrfachen der Letalität der im Rahmen der saisonalen Influenza Infizierten, welche - als Orientierungshilfe - mit einem Wert von 0,1\% geschätzt wird. Für eine Hochrechnung der Mortalität bezogen auf die Bevölkerung insgesamt muss zudem der aktuelle Anteil der tatsächlich Infizierten (spezifische Mortalität) und für eine Vorausberechnung der Anteil der potentiell Empfänglichen berücksichtigt werden. Dieser wird für SARS-CoV-2 ohne Infektionsschutzmaßnahmen, ausgehend von der Basisreproduktionszahl von etwa 3, auf $60-70 \%$ geschätzt und liegt damit etwa 4-mal höher als bei der saisonalen Influenza. In einer konservativen Abschätzung des Impacts auf die Sterblichkeit in der Bevölkerung multipliziert sich somit eine etwa 4-fach höhere individuelle IFR mit einer vermutlich 4-fach höheren Befallsrate („attack rate“) der Bevölkerung auf den Faktor 16 gegenüber der saisonalen Influenza. Hinzu kommen bei COVID-19 noch mögliche Spätfolgen durch einen Multiorganbefall (chronische Morbidität bei Genesenen).

Klinik und Versorgungsforschung, Public Health und Politik Beobachtungen aus größeren klinischen Fallserien werden zunehmend verfügbar und helfen, die klinisch-epidemiologischen Daten abzusichern und Empfehlungen für ein bestmögliches Management zu entwickeln. Aus den diesbezüglichen Erkenntnissen zu einer Beatmungspflichtigkeit lassen sich z. B. Hoch- und Vorausberechnungen zum Bedarf an Beatmungsplätzen im weiteren Pandemieverlauf ableiten. Jedes Jahr sterben in Deutschland etwa 900000 Menschen an allen Todesursachen zusammengenommen. Diese allgemeine jährliche Mortalität von 1 \% könnte bei Nicht-Handeln durch die für COVID-19 mögliche spezifische Mortalität zeitlich befristet um die Hälfte ansteigen und betrifft dabei nicht nur die letzten Lebensmonate schwerkranker Menschen [13]. Aus Public-Health-Perspektive ist im weiteren die epidemiologische Triade von Erreger (Agent), (menschlichem) Wirtsorganismus (Host) und der natürlichen und von Menschen (mit-) gestalteten Umwelt (Environment) des Anthropozäns zu berücksichtigen: Individuelle und soziale Vulnerabilitäten und Verhaltensweisen, ethische Desiderate, die besonderen Verhältnisse institutionalisierter oder anderweitig dicht beieinander lebender Personengruppen sowie wirtschaftliche und soziale Langzeitfolgen sind ebenso von Bedeutung für die gesundheitlichen Folgen wie eine mögliche Carrierfunktion in tiefere Lungenabschnitte von Aerosolen in der Außen- und Innenluft oder auch die Auswirkungen der Gesundheitsschutzmaßnahmen selbst - Epidemien sind ebenso sehr gesellschaftliche Phänomene wie sie biologische Phänomene sind [14-17].

So ist zu hoffen, dass eine Pandemie im 21. Jahrhundert anders verläuft als die in manchen gesellschaftlichen Gruppierungen schon vergessen scheinenden Pandemien vergangener Jahrhunderte, welche mit weniger Wissen(schaft) und weniger gesellschaftlicher Kommunikation mit verheerenden Folgen verliefen [18]. Millionen von Todesfällen und Abermillionen von verlorenen Lebensjahren können heute vermieden werden - wenn Vernunft auf sich selbst vertraut und der bei erfolgreichen Infektionsschutzmaßnahmen wieder unsichtbaren Bedrohung mit den ebenfalls nicht greifbaren menschlichen Fähigkeiten von Klugheit und Humanität anhaltend begegnet wird. Eine Hoffnung, die allerdings nur realistisch ist unter der Bedingung einsichtigen und gezielten individuellen und gesellschaftlichen Handelns. Die historisch belegten Alternativen bei Einsichts- und Tatenlosigkeit, ebenso wie bei therapeutischer und präventiver Erfolglosigkeit sind eine zweite Pandemie-Welle, trotz einer vorübergehenden Beruhigung (,the light at the end of the tunnel might be a train coming“), mehrere kleine „zweite Wellen“ oder ein kontinuierlicher „slow burn“, bei welchem sich das Leiden in der betroffenen Bevölkerung auf mehrere Jahre verteilt [19, 20]. Damit liegt nicht eine Zukunft unabänderlich vor einer Gesellschaft, sondern mehrere, im Vorfeld noch gestaltbare und damit gesell- 
schaftlich zu verhandelnde Zukunftsoptionen. Es ist zu erwarten, dass der oben skizzierte „Worst Case“ kontrafaktisch bleibt, die gesellschaftlich dann letztlich realisierte Zukunftsoption wird allerdings nicht mehr nur optierbar sein. Anzustreben ist dafür eine Situation der bestmöglichen medizinischen Versorgung der jeweils Betroffenen - auch für den Fall des Ausbleibens von effektiven Impfstoffen als das Szenario einer gebremsten Durchinfizierung.

Zu allen genannten Aspekten gibt es einerseits bereits aufschlussreiche Erkenntnisse, andererseits stellen sich noch viele Fragen - im Einzelnen wie in ihrer Gesamtheit. Politische Entscheidungsträger sind in der Pflicht, auch unter unvollständiger Datenlage vernünftige und menschlich verantwortbare Entscheidungen zu treffen und in komplexen Zusammenhängen eine bestmögliche allokative Effizienz begrenzter Ressourcen an Zeit, Personal, Kompetenz und Kapazität mit einer höchstmöglichen Akzeptanz der getroffenen Maßnahmen zu vereinen. Ungerechtfertigter Aktionismus, im diagnostischen, therapeutischen oder präventiven Handlungsfeld verbietet sich ethisch, ökonomisch und politisch. Dass vor diesen Imperativen „old“ Public Health im Sinne eines technisch und konzeptionell modernisierten Gesundheitsschutzes und „new“ Public Health im Sinne proaktiver systemischer Ansätze der Gesundheitsförderung zusammenfinden sollten, kann nur ein um das andere Mal wiederholt werden [21]. Gerade in einem Global Health-Kontext hätten international vernetzte Schools of Public Health dazu beitragen können, die notwendigen Theorien und Ethiken für die Public-Health-Praxis über die Ländergrenzen hinweg bereitzustellen: Manches hätte sich bei der COVID-19-Pandemie dadurch international womöglich schon im Ansatz verhindern lassen.

Eine notwendige und unverzichtbare, situationsangemessene Komplexität des Handelns wollen auch die Beiträge in diesem Heft in wieder verschiedenen Bereichen unterstützen. Bei aller gerechtfertigten Sorge hinsichtlich der Pandemiefolgen dürfen die Belastungen nicht unbalanciert zu Lasten anderer Patientengruppen gehen. Die Themen sind daher nicht nur Mitarbeiterinfektionsschutz in Zeiten der Coronavirus-Pandemie durch telemedizinische Mitarbeiterkonferenzen, ein Cochrane Rapid Review zur Quarantäne alleine oder in Kombination mit weiteren Public-Health-Maßnahmen und eine Covid-19-bezogene Public-Health-Ethikberatung, sondern auch neue Formen der Zusammenarbeit im ambulanten und stationären Sektor, motivationale Faktoren für die Präferenz der Fachrichtung Allgemeinmedizin unter Medizinstudierenden NRWs, Determinanten der Niederlassung als Allgemeinmediziner, die Evidenzbasierung der Medikamentenverordnungen in Deutschland, Facebook-Bewertungen zu Versorgungsqualität und Patientenzufriedenheit in Krankenhäusern, sektorenübergreifende Entwicklungen in der Notfallversorgung und der Impact von Änderungen der Vergütung auf den Erfolg der Behandlung in psychiatrischen Kliniken.

Um am Ende noch einmal auf den Anfang zu kommen: Gottlob Benjamin Jäsche fährt in seinem Handbuch zu Immanuel Kants Logik-Vorlesung fort: „Der Philosoph muß also bestimmen können 1) die Quellen des menschlichen Wissens, 2) den Umfang des möglichen und nützlichen Gebrauchs alles Wissens und endlich 3) die Grenzen der Vernunft."[1]. Eine Skepsis bzgl. der Realität der möglichen Ereignisschwere in Teilen der Bevölkerung mag am Anfang der Pandemie eine durchaus vernünftige Position gewesen sein - vor dem
Hintergrund der zwischenzeitlich erarbeiteten, auch erlittenen und in ihren Quellen belegbaren Erkenntnisse wäre sie unvernünftig. Eine nun erneut zu beobachtende Skepsis vor dem Hintergrund erfolgreicher Schutzmaßnahmen ist ein für den Public-Health-Bereich typisches Phänomen und hat in der Vergangenheit zur teilweise gefährlichen Vernachlässigung kritischer Infrastruktur in den Public-Health-Aufgabenfeldern von Hygiene und ÖGD und auch der benachbarten präventiven Handlungsfelder der Arbeits- und Sozialmedizin geführt. Die aktuell fehlenden Lehrstühle in diesen Bereichen sind Indikatoren dieser kritischen Entwicklung und fehlen zudem als demokratische Absicherungen in Katastrophenlagen mit zeitweise beschränkten bürgerlichen Freiheiten. Solche Lehrstühle wären zudem geeignete Instrumente, um differenziert und jeweils angemessen auf die Bedrohungslage reagieren zu können - sie wären auch hoch rentable Investitionen angesichts der großen ökonomischen Auswirkungen der Pandemie-Bekämpfungsmaßnahmen.

Auf die Frage „Was kann ich wissen?“ folgt nicht nur akademisch die Frage „Was soll ich tun?“. Der bei Tatenlosigkeit bzw. verspätetem Handeln mögliche Pandemieverlauf gegenüber dem durch einsichtige, vernünftige Infektionsschutzmaßnahmen faktischen Verlauf wird im Ländervergleich augenfällig (siehe u. a. euromomo. eu). Die kommunikative Herausforderung nimmt bei anhaltender Skepsis und günstiger Lage allerdings zu und verlangt paradoxerweise gerade im Erfolgsfall eine noch einmal höhere Rationalität und Begründbarkeit des Handelns für das Bestehen einer über kurz oder lang gesellschaftlich geforderten Rechenschaftslegung. Angezeigt ist daher ein „nützlicher Gebrauch alles Wissens“ in Form von flexiblen, der Lage und den jeweiligen Bevölkerungsgruppen angepassten und aufeinander abgestimmten modularen Maßnahmenbündeln. Die noch offenen Fragen sind zahlreich, das NichtWissen ist immer größer als das Wissen. Investitionen in Forschung und Entwicklung sind unabdingbar und ein Gebot gesundheitspolitischer wie auch ökonomischer Klugheit. Dass letztlich auch die Vernunft ihre Grenzen hat, dürfte wiederum paradoxerweise der Narr und der Weise sprechen, Gottlob Benjamin Jäsche und Immanuel Kant sei's geklagt.

\section{Interessenkonflikt}

Die Autorinnen/Autoren geben an, dass kein Interessenkonflikt besteht.

\section{Literatur}

[1] Jäsche GB. Immanuel Kants Logik - ein Handbuch zu Vorlesungen. Königsberg, Friedrich Nicolovius. 1800; URL: https://www.textlog.de/ kant-logik-philosophie-0.html, download 24.05.2020

[2] Poser H. Wissenschaftstheorie: Eine philosophische Einführung. Stuttgart, Reclam; 2001

[3] Kant I. Prolegomena zu einer jeden künftigen Metaphysik, die als Wissenschaft wird auftreten können. 1783; URL: https://korpora.zim. uni-duisburg-essen.de/kant/aa04/ S 260, download 24.05.2020

[4] Box, GEP Science and statistics. J Am Statist Ass. 1976; 71: 791-799. URL: http://www-sop.inria.fr/members/lan.Jermyn/philosophy/ writings/Boxonmaths.pdf. download 24.05.2020 
[5] Deutsches Ärzteblatt. Genom-Analysen klären Herkunft von 2019-nCoV (30.01.2020). URL: https://www.aerzteblatt.de/ nachrichten/109051/Genom-Analysen-klaeren-Herkunft-von2019-nCoV, download 24.05.2020

[6] Deutsches Ärzteblatt. COVID-19: Befall der Endothelien könnte Multiorganversagen erklären (20.04.2020). URL: https://www. aerzteblatt.de/nachrichten/112129/COVID-19-Befall-der-Endothelienkoennte-Multiorganversagen-erklaeren, download 24.05.2020

[7] Wölfel R, Corman VM, Guggemos W et al. Virological assessment of hospitalized patients with COVID-2019. Nature 2020; URL: https:// www.nature.com/articles/s41586-020-2196-x, download 24.05.2020

[8] Wong JY, Kelly $\mathrm{H}$, Ip DKM et al. Case fatality risk of influenza A(H1N1pdm09): a systematic review. Epidemiology 2013; 24. doi:10.1097/EDE.0b013e3182a67448

[9] Gianicolo E, Riccetti N, Blettner M. et al. Epidemiologische Maßzahlen im Rahmen der COVID-19-Pandemie. Dtsch Arztebl Int 2020; 117: 336-342

[10] Robert Koch-Institut. SARS-CoV-2 Steckbrief zur Coronavirus-Krankheit-2019 (COVID-19) (Stand: 22.5.2020). URL: https://www.rki.de/ DE/Content/InfAZ/N/Neuartiges_Coronavirus/Steckbrief.html. download 24.05.2020

[11] Ghani AC, Donnelly CA, Cox DR et al. Methods for Estimating the Case Fatality Ratio for a Novel, Emerging Infectious Disease. Am J Epidemiol 2005; 162: 479-486

[12] Streeck H, Schulte B, Kümmerer BM et al. Infection fatality rate of SARS-CoV-2 infection in a German community with a super-spreading event. URL: https://www.ukbonn.de/C12582D3002FD21D/vwLookupDownloads/Streeck_et_al_Infection_fatality_rate_of_SARS_CoV_2_infection2.pdf/\%24FILE/Streeck_et_al_Infection_fatality_rate_of_SARS_ CoV_2_infection2.pdf. download 26.05.2020

[13] Hanlon P, Chadwick F, Shah A et al. COVID-19 - exploring the implications of long-term condition type and extent of multimorbidity on years of life lost: a modelling study [version 1; peer review: awaiting peer review]. Wellcome Open Res 2020; 5: 75
[14] Viner RM, Mytton OT, Bonell C et al. Susceptibility to SARS- CoV-2 infection amongst children and adolescents compared with adults: a systematic review and meta-analysis. URL: https://www.medrxiv.org/ content/10.1101/2020.05.20.20108126v1.full.pdf. download 24.05.2020

[15] Wu X, Nethery RC, Sabath BM et al. Exposure to air pollution and COVID-19 mortality in the United States: A nationwide cross-sectional study. https://www.medrxiv.org/content/10.1101/2020.04.05.200545 02v2, download 24.05.2020

[16] Kawohl W, Nordt C. COVID-19, unemployment, and suicide. URL: https://www.thelancet.com/action/showPdf?pii = S22150366\%2820\%2930141-3, download 24.05.2020

[17] Pandemiebewältigung und nachhaltige Verbesserung der Gesundheit der Bevölkerung. Stellungnahme des Zukunftsforums Public Health zur COVID-19-Pandemie (19.05.2020). URL: https://zukunftsforum-public-health.de/stellungnahme-covid/, download 26.05.2020

[18] Kolata G. Flu: The Story of the Great Influenza Pandemic of 1918 and the Search for the Virus That Caused It. New York: Touchstone; 1999

[19] Ferguson N, Laydon D, Nedjati-Gilani G et al. Report 9: Impact of non-pharmaceutical interventions (NPIs) to reduce COVID-19 mortality and healthcare demand (16.03.2020). URL: https://www. imperial.ac.uk/media/imperial-college/medicine/mrc-gida/202003-16-COVID19-Report-9.pdf. download 26.05.2020

[20] Moore KA, Lipsitch M, Barry JM et al. The Future of the COVID-19 Pandemic: Lessons Learned from Pandemic Influenza (30.04.2020). URL: https://www.cidrap.umn.edu/sites/default/files/public/ downloads/cidrap-covid19-viewpoint-part1_0.pdf. download 26.05.2020

[21] Wildner M, Wieler LH, Zeeb $\mathrm{H}$. for the standing committee of the Future Forum Public Health. Germany's expanding role in global health. Lancet 2018; 391: 657 